\title{
Disk growth in bulge-dominated galaxies: molecular gas and morphological evolution
}

\author{
Lisa Young ${ }^{1}$, Martin Bureau ${ }^{2}$, Alison Crocker $^{2}$ and \\ Francoise Combes ${ }^{3}$ \\ ${ }^{1}$ Physics Department, New Mexico Tech, Socorro, NM 87801, USA \\ email: lyoung@physics.nmt.edu \\ ${ }^{2}$ Sub-department of Astrophysics, University of Oxford, Keble Road, Oxford, OX1 3RH, UK \\ ${ }^{3}$ Obervatoire de Paris, LERMA, 61 Av. de l'Observatoire, 75014, Paris, France
}

\begin{abstract}
Substantial numbers of morphologically regular early-type (elliptical and lenticular) galaxies contain molecular gas, and the quantities of gas are probably sufficient to explain recent estimates of the current level of star formation activity. This gas can also be used as a tracer of the processes that drive the evolution of early-type galaxies. For example, in most cases the gas is forming dynamically cold stellar disks with sizes in the range of hundreds of pc to more than one kpc, although there is typically only $1 \%$ of the total stellar mass currently available to form young stars. The numbers are still small, but the molecular kinematics indicate that some of the gas probably originated from internal stellar mass loss while some was acquired from outside. Future studies will help to quantify the role of molecular gas (dissipational processes) in the formation of early-type galaxies and their evolution along the red sequence.
\end{abstract}

Keywords. galaxies: elliptical and lenticular, cD - galaxies: ISM - galaxies: evolution galaxies: individual (NGC 2768, NGC 3032, NGC 3656, NGC 4150, NGC 4459, NGC 4526)

\section{The Molecular Gas Content of Early-type Galaxies}

About a decade ago, surveys for molecular gas in early-type galaxies began to show significant detection rates (e.g. Lees et al. 1991; Wiklind et al. 1995; Knapp \& Rupen 1996). The difficulty was that the surveys had very strong selection biases towards galaxies that are bright at $100 \mu \mathrm{m}$, and it was virtually impossible to know how to extrapolate to FIR-faint galaxies. In recent years this limitation has been removed and there are now deeper surveys of less biased samples. Welch \& Sage (2003) found a surprisingly high $\mathrm{CO}$ detection rate of $78 \%$ in a volume-limited sample of nearby field lenticular galaxies, and Sage et al. (2007) detected CO emission in 33\% of a similar sample of field ellipticals. Combes et al. (2007) also detected CO emission in $28 \%$ of the early-types in the SAURON survey (de Zeeuw et al. 2002), which is not a complete sample in any sense but has the advantage that the gas content can be compared to information on the stellar populations, internal dynamics, and ionized gas. In short, when the samples are not FIRbiased there are still a significant number of early-type galaxies showing CO emission. Cold gas masses are highly variable however, with $M_{\text {gas }} / L_{B}$ in the range $10^{-1}$ to $10^{-3}$ and lower.

The strongest connection between cold gas and galaxy properties is as expected with indicators of recent star formation, including stronger $\mathrm{H} \beta$ emission and absorption (Combes et al. 2007). Lucero \& Young (2007) also discuss the connections between molecular gas, star formation, radio continuum and FIR emission, and the CO detection rate in earlytypes is at least superficially consistent with the incidence of star formation derived from 
GALEX UV data (e.g. Yi et al. 2007; Kaviraj et al. 2007). Thus, the present gas content of early-type galaxies does clearly reveal the potential for star formation and disk growth.

\section{Disk Growth}

The morphological changes that can be effected in early-type galaxies by star formation, and the ease with which feedback can disrupt it, will all depend on the distribution of the molecular gas. A few early-types have now had their CO emission mapped by millimeter interferometers, e.g. Inoue et al. (1996), Wiklind et al. (1997), Young (2002, 2005), Okuda et al. (2005), Das et al. (2005), and Young et al. (2008). The molecular gas is typically found in regular, kpc-scale rotating disks with masses of a few $10^{7}$ to a few $10^{9} \mathrm{M}_{\odot}$ of molecular hydrogen. The disk radii range from a tenth up to a few effective radii of the stellar distribution. The average surface densities are often $100 \mathrm{M}_{\odot} \mathrm{pc}^{-2}$ and higher, comparable to the molecular surface densities in spirals.

When both molecular maps and two-dimensional optical spectroscopy are available, there is usually good evidence for a young stellar disk coincident with the molecular disk. The most dramatic example is the Virgo cluster lenticular NGC 4526 (see Fig. 1; Young et al. 2008), which has embedded in its bulge a dynamically cold kpc-scale stellar disk with a substantially younger mean age than the rest of the bulge (Emsellem et al. 2004; Kuntschner et al. 2006). The $[\mathrm{O}$ III $] / \mathrm{H} \beta$ line ratios are also suggestive of current star formation activity (Sarzi et al. 2006) and the ionized gas kinematics follow the molecular kinematics, albeit at somewhat smaller velocity amplitude. Based on the FIR flux, the star formation rate in NGC 4526 is roughly $1 \mathrm{M}_{\odot} \mathrm{yr}^{-1}$, giving a gas depletion timescale of $7 \times 10^{8} \mathrm{yr}$. But the total molecular mass now present in the galaxy is only $0.6 \%$ of the total stellar mass, so that even if there was substantially more molecular gas in the recent past it can not make more than a small frosting of young stars. The Virgo lenticular NGC 4459 is a similar case (Young et al. 2008).

\section{The Origin of the Cold Gas}

For at least 30 years it has been speculated that early-type galaxies should have a substantial cold ISM originating in mass loss from their own evolved stars (Faber \& Gallagher 1976). A modern version of the theory, complete with heating, cooling, a proper cosmological context and the influence of AGN can be found in Temi et al. (2007) and references therein. It is also well known that mergers and the accretion of satellites and/or cold gas can be important mechanisms for galaxy growth. The relative importance of internal and external gas sources for early-type galaxies is not yet well known, but angular momentum can provide a strong constraint since internally produced gas must have a specific angular momentum consistent with that of the stars in the galaxy.

Sarzi et al. (2006) have measured the distribution of the misalignment angle between the stellar and ionized-gas kinematic major axes for the SAURON sample. They find evidence for ionized gas from both internal origins (a large peak of galaxies with no misalignment) and external origins (a long tail of misalignments out to $180^{\circ}$, counterrotation). The corresponding data for molecular gas are much more rare, but appear to be consistent with the above picture (see also Bureau \& Chung 2006). Of the four lenticulars mapped by Young et al. (2008), one (NGC 3032) has molecular gas counterrotating with respect to the stars, so this gas must be left over from a major merger or acquired from an external source. In the obvious merger remnant NGC 3656, the gas disk is roughly perpendicular to the stellar kinematic major axis (Young 2002), and in NGC 2768 the gas forms a polar ring (or disk) structure (Crocker et al. 2008), but much more commonly the 
gas disks are aligned with the mean stellar rotation and suggest a contribution from internal mass loss. NGC 4150 harbours a counter-rotating young stellar core, but strangely the $\mathrm{CO}$ gas is rotating like the galaxy bulk (Young et al. 2008).

The misaligned molecular disks in NGC 3032 and in NGC 3656 are both massive, kpc-scale, and vigorously forming stars. Clearly, even when the molecular gas has an external origin, it does not always descend into the nucleus of the galaxy to feed and/or be destroyed by an active nucleus. A better global understanding of the influence of the accreted cold gas on early-type galaxies therefore requires an accurate model for the impact parameters of this gas and the size distribution of the resulting gas disks.

\section{Open Questions}

We have seen that kpc-scale molecular disks with $10^{8}$ to $10^{9} \mathrm{M}_{\odot}$ of molecular gas are relatively common in early-type galaxies at $z=0$, and the incidence of these disks is probably consistent with current star formation rate estimates based on UV data. Environmental effects on the gas content of early-type galaxies have not been properly explored yet, nor have the possible effects of AGN activity to clear cold gas. The observed molecular disks clearly document the process of disk growth within bulges, a process which must have been of greater importance in the past, but the details remain sketchy.

It is not yet known whether the molecular gas always forms stars (and whether that result is consistent with our theoretical understanding of star formation processes). It has been hypothesized that molecular disks should be stabilized by the galaxies' steep gravitational potentials (e.g. Kennicutt 1989; Okuda et al. 2005; Kawata et al. 2007). Clearly our data have shown that the molecular gas is often associated with current star formation, but perhaps this is not always the case. In NGC 2768 the central molecular disk (only $\approx 200 \mathrm{pc}$ ) is located in a prominent drop in the stellar velocity dispersion, just as one would expect if a dynamically cold stellar disk had formed (Crocker et al. 2008). Curiously, however, there is no corresponding $\mathrm{H} \beta$ absorption feature which would suggest current or recent star formation. Early-type galaxies may thus present a valuable testing ground for galaxy-scale theoretical models of star formation.

Another open question has to do with the role of gas in the formation of early-type galaxies in general, and more specifically in the origin of the kinematically distinct substructures that are so common in early-type galaxies (e.g. McDermid et al. 2006). The SAURON galaxies contain a large variety of cold stellar disks (some of them metalenriched) and misaligned or counterrotating cores, in many sizes, and surely these are important clues to the galaxy formation processes. It would be useful to know what fraction of the substructures (or which kinds) are formed through dissipationless processes and which required gas. The answer to this question will likely require studies of more complete samples, such as Atlas ${ }^{3 \mathrm{D}}$.

Nevertheless, very preliminary indications are that the molecular gas probably originated in a combination of internal stellar mass loss and cold accretion from external sources. Again, the available numbers are still small, and a more quantitative assessment requires a substantial increase in the number of galaxies with both cold gas and stellar kinematic maps.

\section{Acknowledgements}

L.Y. is partially supported by NSF AST-0507432, and is indebted to all the members of the galaxy group at the University of Oxford for welcoming her into such a stimulating environment during her recent sabbatical. 

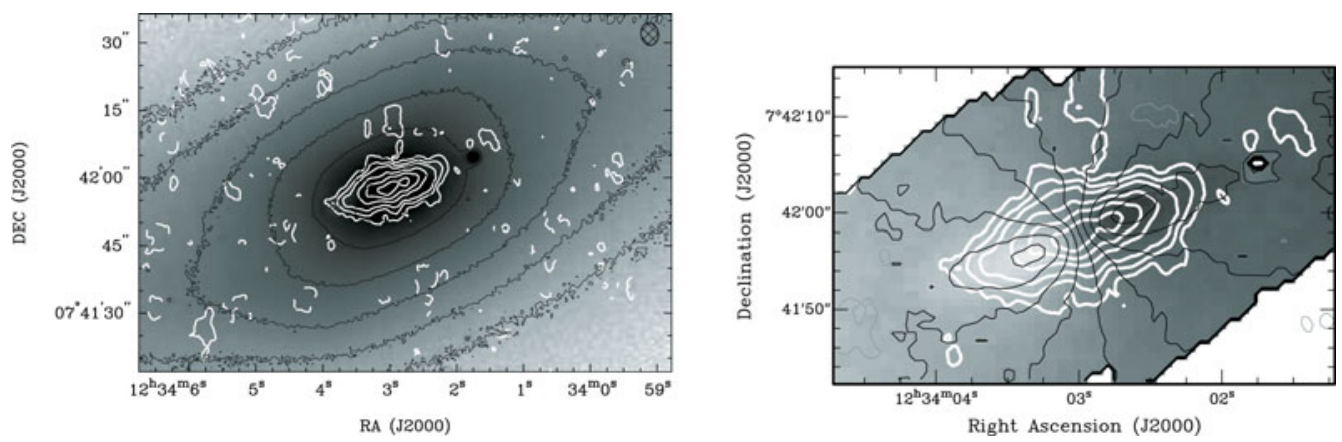

Figure 1. Integrated CO intensity in NGC 4526 (white contours), overlaid on respectively a SDSS $g$ image (left) and the stellar velocity field (right; greyscale and black contours; Young et al. 2008). CO contours are at $-10,10,20,30,50,70$, and $90 \%$ of the peak $\left(37 \mathrm{Jy} \mathrm{beam}^{-1} \mathrm{~km} \mathrm{~s}^{-1}\right)$. Velocity contours are from 416 to $816 \mathrm{~km} \mathrm{~s}^{-1}$ in intervals of $50 \mathrm{~km} \mathrm{~s}^{-1}$.

\section{References}

Bureau, M. \& Chung, A. 2006, MNRAS, 366, 182

Combes, F., Young, L. M., \& Bureau, M. 2007, MNRAS, 377, 1795

Crocker, A. F., Bureau, M., Young, L. M., \& Combes, F., MNRAS, submitted

Das, M., Vogel, S. N., Verdoes Kleijn, G. A., O’Dea, C. P., \& Baum, S. A. 2005, ApJ, 629, 757 de Zeeuw, P. T., et al. 2002, MNRAS, 329, 513

Emsellem, E., et al. 2004, MNRAS, 352, 721

Faber, S. M. \& Gallagher, J. S. 1976, ApJ, 204, 365

Inoue, M. Y., Kameno, S., Kawabe, R., \& Inoue, M. 1996, AJ, 111, 1852

Kaviraj, S., Schawinski, K., Devriendt, J. E. G., et al. 2006, ApJ, submitted (astro-ph/0601029)

Kawata, D., Cen, R., \& Ho, L. C. 2007, ApJ, in press (astro-ph/0706.0005)

Kennicutt, R. C., Jr. 1989, ApJ, 344, 685

Knapp, G. R. \& Rupen, M. P. 1996, ApJ, 460, 271

Kuntschner, H., Emsellem, E., Bacon, R., Bureau, M., et al. 2006, MNRAS, 369, 497

Lees, J. F., Knapp, G. R., Rupen, M. P., \& Phillips, T. G. 1991, ApJ, 379, 177

Lucero, D. M., \& Young, L. M. 2007, AJ, in press (astro-ph/0708.3873)

McDermid, R. M., Emsellem, E., Shapiro, K. L., et al. 2006, MNRAS, 373, 906

Okuda, T., Kohno, K., Iguchi, S., \& Nakanishi, K. 2005, ApJ, 620, 673

Sage, L. J., Welch, G. A., \& Young, L. M. 2007, ApJ, 657, 232

Sarzi, M., Falcón-Barroso, J., Davies, R. L., Bacon, R., et al. 2006, MNRAS, 366, 1151

Temi, P., Brighenti, F., \& Mathews, W. G. 2007, ApJ, 660, 1215

Welch, G. A. \& Sage, L. J. 2003, ApJ, 584, 260

Wiklind, T., Combes, F., \& Henkel, C. 1995, A\&A, 297, 643

Wiklind, T., Combes, F., Henkel, C., \& Wyrowski, F. 1997, A\&A, 323, 727

Yi, S. K., Yoon, S.-J., Kaviraj, S., et al. 2005, ApJ, 619, L111

Young, L. M. 2002, AJ, 124, 788

Young, L. M. 2005, ApJ, 634, 258

Young, L. M., Bureau, M., \& Cappellari, M. 2008, ApJ, in press (astro-ph/0712.4189) 